\title{
Genetic testing for Mendelian stroke due to cerebrovascular anomalies and other syndromes
}

\author{
Paolo Enrico Maltese ${ }^{1 \star}$, Yeltay Rakhmanov ${ }^{1}$, Alice Bruson ${ }^{1}$, Lorenzo Lorusso ${ }^{2}$ and Matteo Bertelli ${ }^{1,3}$
}

\begin{abstract}
Stroke is defined as a focal or at times global neurological impairment of sudden onset and presumed vascular origin. 85\% of strokes are due to cerebral ischemia and the other $15 \%$ to primary intracerebral hemorrhage.

Ischemic stroke (IS) is characterized by complete or partial obstruction of a vessel in the brain, resulting in lack of blood supply and death of brain tissue. The most common causes of IS are atherosclerosis, cardioembolism and small-vessel disease (lacunar stroke). Genetic factors play important role. Incidence rates for IS in the 15 - to 45 -year age range are $\approx 10$ per 100,000 person years.

Hemorrhagic stroke (HS) is the least treatable and the most fatal form of cerebrovascular disease. Genetic mechanisms play a role in its development. Occurrence depends on many risk factors, including hypertension, heavy alcohol intake and anticoagulant treatment. According to the World Health Organization, 15 million people suffer stroke worldwide each year. The overall incidence of spontaneous HS worldwide is 24.6 per 100,000 person years. Strokes are the third most common cause of death and the most common cause of disability in developed countries.

This Utility Gene Test was developed on the basis of an analysis of the literature and existing diagnostic protocols. It is useful for confirming diagnosis, as well as for differential diagnosis, couple risk assessment and access to clinical trials.
\end{abstract}

Keywords: Ischemic stroke, hemorrhagic stroke, EBTNA UTILITY GENE TEST

'MAGI's Lab, Rovereto, Italy

${ }^{2}$ U.O.C. di Neurologia, A.S.S.T. della Franciacorta, Chiari (BS), Italy

${ }^{3}$ MAGI Euregio, Bolzano, Italy

*Corresponding author: P. E. Maltese E-mail: paolo.maltese@assomagi.org DOI: 10.2478/ebtj-2018-0045
Mendelian stroke due to cerebrovascular anomalies and other syndromes (Other synonyms: Cerebral infarction)

\section{General information about the disease}

The World Health Organization defines stroke as a focal (or at times global) neurological impairment of sudden onset, lasting more than $24 \mathrm{~h}$ (or leading to death) and of presumed vascular origin (1). $85 \%$ of strokes are due to cerebral ischemia and $15 \%$ to intracerebral hemorrhage $(2,3)$. Stroke is the third leading cause of death and the most common cause of disability in developed countries.

Genetic causes of stroke range from Mendelian to complex factors.

This Utility Gene Test is focused on rare Mendelian forms of stroke. Mendelian stroke can be caused by a number of monogenic disorders, and in such cases is frequently part of a multisystem disorder.

\section{Ischemic stroke}

Ischemic stroke (IS) is a condition caused by complete or partial obstruction of a vessel in the brain, resulting in lack of blood supply and death of brain tissue. Genetic factors play an important role in the development of IS. Studies on twins have demonstrated that stroke prevalence is about five times higher in monozygotic than in dizygotic twins (4). (c) 2018 Authors. This work was licensed under the Creative Commons AttributionNonCommercial-NoDerivs 3.0 License. 
In a large group of stroke patients, the odds ratio of having a family history of stroke was 2.24 for large vessel-disease and 1.93 for small vessel disease (5). For stroke patients under the age of 65 , the odds ratio was even higher: 2.93 and 3.15, respectively $(5,6)$.

The most common causes of IS are atherosclerosis, cardioembolism and small-vessel disease (lacunar stroke) (2). Non-modifiable risk factors (age, African or Asian ethnicity, male sex) and acquired risk factors (hypertension, cigarette smoking, diabetes, atrial fibrillation and obesity) account for much of the risk of ischemic stroke (7). However, the underlying mechanisms of most of these risk factors are genetic.

Incidence rates for IS in the 15 to 45 -year age range are $\approx 10$ per 100,000 person years for individuals of predominantly European ancestry, with similar rates for men and women (8).

IS occurs suddenly and may have the following symptoms, depending on the affected area of the brain: unilateral insensitivity or paralysis, confusion and difficulty speaking or following speech, dizziness, loss of balance, coordination, abnormal migraine accompanied by facial pain, sudden reduction or loss of vision, vomiting and altered states of consciousness.

Diagnosis is based on routine physical examination, neurological examination, non-contrast computerized tomography, CT angiography, MR imaging and genetic testing.

Differential diagnosis should consider acute hypoglycemia, brain neoplasms, encephalitis headache, migraine, hypernatremia in emergency medicine, hyperosmolar hyperglycemic nonketotic coma, hypertensive emergencies, hyponatremia, labyrinthitis ossificans, meningitis, hemorrhagic stroke, subarachnoid hemorrhage, subdural hematoma and transient ischemic attack.

Familial and sporadic cases of Mendelian IS are welldocumented in the literature and show a greater incidence at young age.

\section{Autosomal dominant syndromic IS}

- Moyamoya disease 5 (MYMY5, OMIM disease 614042) ACTA2 (OMIM gene 102620);

- Angiopathy, hereditary, with nephropathy, aneurysms, and muscle cramps (HANAC, OMIM disease 611773); Brain small vessel disease with or without ocular anomalies (BSVD, OMIM disease 607595); Porencephaly 1 (POREN1, OMIM disease 175780) - COL4A1 (OMIM gene 120130).

- Cerebral arteriopathy with subcortical infarcts and leukoencephalopathy 1 (CADASIL1, OMIM disease 125310) - NOTCH3 (OMIM gene 600276).

\section{Other likely genes}

- COL4A2 (OMIM gene 120090) (9).

\section{Autosomal recessive syndromic IS}

- Homocystinuria, B6-responsive and nonresponsive types (OMIM disease 236200) - CBS (OMIM gene 613381);

- Polyarteritis nodosa, childhood-onset (PAN, OMIM disease:
615688) - CECR1 (OMIM gene 607575);

- Moyamoya 6 with achalasia (MYMY6, OMIM disease 615750) - GUCY1A3 (OMIM gene 139396);

- Cerebral arteriopathy, autosomal recessive, with subcortical infarcts and leukoencephalopathy (CARASIL, OMIM disease 600142) - HTRA1 (OMIM gene 602194);

- Homocystinuria due to deficiency of $\mathrm{n}(5,10)$ methylenetetrahydrofolate reductase activity (MTHFR) deficiency (OMIM disease 236250) - MTHFR (OMIM gene 607093)

- Congenital disorder of glycosylation, type Ia (CDG1A, OMIM disease 212065) - PMM2 (OMIM gene 601785)

- Aicardi-Goutieres syndrome-5 (AGS5, OMIM disease 612952) - SAMHD1 (OMIM gene 606754) (10).

\section{$\mathrm{X}$-linked syndromic IS}

- Fabry disease (OMIM disease 301500) - GLA (OMIM gene 300644).

\section{Mitochondrial syndromic IS}

- Mitochondrial myopathy, encephalopathy, lactic acidosis and stroke-like episodes (MELAS, OMIM disease 540000) MT-TL1 (OMIM gene 590050) and MT-ND5 (OMIM gene 516005).

\section{Autosomal dominant and recessive syndromic IS}

- Aicardi-Goutieres syndrome 1, dominant and recessive (AGS1, OMIM disease 225750) - TREX1 (OMIM gene 606609).

Pathogenic variants may include missense, nonsense, splicing, small insertions and deletions, small indels, gross deletions and duplications.

\section{Hemorrhagic stroke}

Hemorrhagic stroke (HS) is an acute manifestation of common progressive cerebrovascular disease among elderly patients. HS is the least treatable and leading cause of acquired disability and mortality. Genetic mechanisms play an important role in its development. It is therefore necessary to develop HS-specific therapeutics and effective primary prevention strategies. Identification of HS-related genes is a promising direction in novel HS prevention (11). Study of genetic mechanisms of HS risk factors, including hypertension, heavy alcohol intake and anticoagulant treatment, is also important (12).

The underlying mechanism of HS is thought to be leakage from small intracerebral arteries damaged by chronic hypertension, bleeding diatheses, iatrogenic anticoagulation, cerebral amyloidosis and cocaine abuse.

HS mostly affects brain sites such as the thalamus, putamen, cerebellum and brainstem. The brain may be damaged by the hemorrhage or by increased intracranial pressure due to a hematoma (13).

According to the World Health Organization, 15 million people suffer stroke worldwide each year. Of these, 5 million 
die and another 5 million are left permanently disabled (14). The overall incidence of spontaneous HS worldwide is 24.6 per 100000 person-years. The 30 -day mortality rate ranges from $35 \%$ to $52 \%$ with only $20 \%$ of survivors expected to have full functional recovery at 6 months. Approximately half of this mortality occurs within the first 24 hours (15-18).

HS is characterized by a relatively sudden onset of symptoms and neurological deficits, the type and severity of which vary according to lesion type, location and size (19). Findings such as coma, headache, vomiting, seizures, neck stiffness and raised diastolic blood pressure increase the likelihood of HS compared to ischemic stroke, but only neuroimaging can provide a definitive diagnosis (20). Focal neurological deficits present in the following ways: hemiparesis, hemisensory loss, visual field cut on the opposite side to the affected hemisphere, gaze preference on the same side as the affected brain, aphasia, unilateral neglect.

Diagnosis is based on routine physical examination, neurological examination, noncontrast computerized tomography, CT angiography, MR imaging and genetic testing.

Differential diagnosis should consider acute hypoglycemia, brain neoplasms, encephalitis headache, migraine, hypernatremia in emergency medicine, hyperosmolar hyperglycemic nonketotic coma, hypertensive emergencies, hyponatremia, labyrinthitis ossificans, meningitis, stroke, ischemic subarachnoid hemorrhage, subdural hematoma, transient ischemic attack.

HS has autosomal dominant and autosomal recessive inheritance.

\section{Autosomal dominant syndromic HS}

- Moyamoya disease 5 (MYMY5, OMIM disease 614042) and thoracic aortic aneurysm-6 (AAT6, OMIM disease 611788) - ACTA2 (OMIM gene 102620);

- Telangiectasia, hereditary hemorrhagic, type 2 (HHT2, OMIM disease 600376) - ACVRL1 (OMIM gene 601284);

- Cerebral amyloid angiopathy, APP-related (CAA, OMIM disease 605714) - APP (OMIM gene 104760);

- Ehlers-Danlos syndrome vascular-type (EDSVASC, OMIM disease 130050) - COL3A1 (OMIM gene 120180);

- Angiopathy, hereditary, with nephropathy, aneurysms, and muscle cramps (HANAC, OMIM disease 611773); Brain small vessel disease with or without ocular anomalies (BSVD, OMIM disease 607595); Porencephaly 1 (POREN1, OMIM disease 175780) - COL4A1 (OMIM gene 120130);

- Porencephaly-2 (POREN2, OMIM disease 614483) COL4A2 (OMIM gene 120090);

- Cerebral amyloid angiopathy (CAA, OMIM disease 105150) - CST3 (OMIM gene 604312);

- Telangiectasia, hereditary hemorrhagic, type 1 (HHT1, OMIM disease 187300) - ENG (OMIM gene 131195);

- Marfan syndrome (MFS, OMIM disease 154700) - FBN1 (OMIM gene 134797);

- Neurofibromatosis, type 1 (NF1, OMIM disease 162200) NF1 (OMIM gene 613113);
- Juvenile polyposis/hereditary hemorrhagic telangiectasia syndrome (JPHT, OMIM disease 175050) - SMAD4 (OMIM gene 600993);

- Amyloidosis, hereditary, transthyretin-related (OMIM disease 105210) - TTR (OMIM gene 176300).

Dominant or paradominant (due to second-hit germline + somatic variation) syndromic HS

- Cerebral cavernous malformations-1 (CCM1, OMIM disease 116860) - KRIT1 (OMIM gene 604214);

- Cerebral cavernous malformations-2 (CCM2, OMIM disease 603284) - CCM2 (OMIM gene 607929);

- Cerebral cavernous malformations-3 (CCM3, OMIM disease 603285) - PDCD10 (OMIM gene 609118).

\section{Autosomal recessive syndromic HS}

- Pseudoxanthoma elasticum (PXE, OMIM disease 264800) ABCC6 (OMIM gene 603234);

- Factor VII deficiency (OMIM disease 227500) - F7 (OMIM gene 613878);

- Moyamoya 6 with achalasia (MYMY6, OMIM disease 615750) - GUCY1A3 (OMIM gene 139396);

- Hemorrhagic destruction of the brain, subependymal calcification, and cataracts (HDBSCC, OMIM disease 613730) - JAM3 (OMIM gene 606871);

- Arterial tortuosity syndrome (ATS, OMIM disease 208050) - SLC2A10 (OMIM gene 606145).

\section{Other likely genes}

- APOE (OMIM gene 107741) (21);

- PMF1/SLC25A44 (OMIM genes 609176/610824) (21);

- FOXC1 (OMIM gene 601090) (22).

Pathogenic variants may include missense, nonsense, splicing, small insertions and deletions, small indels and gross deletions and duplications.

\section{Aims of the test}

- To determine the gene defect responsible for the disease;

- To confirm clinical diagnosis;

- To assess the recurrence risk and perform genetic counselling for at-risk/affected individuals.

\section{Test characteristics}

\section{Expert centers/ Published Guidelines}

The test is listed in the Orphanet database and is offered by 39 accredited medical genetic laboratories in the EU, and in the GTR database, offered by 17 accredited medical genetic laboratories in the US.

Guidelines for clinical use of the test are described in Genetics Home Reference (ghr.nlm.nih.gov).

\section{Test strategy}

Clinically distinguishable syndromes can be analyzed by sequencing only those genes known to be associated with that 
specific disease using Sanger or Next Generation Sequencing (NGS); if the results are negative, or more generally if clinical signs are ambiguous for diagnosis, a multi-gene NGS panel is used to detect nucleotide variations in coding exons and flanking introns of the above genes.

The test for paradominant genes (KRIT1, CCM2 or PDCD10) is to compare results obtained from germinal lineage (blood or saliva specimens) and affected tissue. For variant selection, a cut-off value (related to biopsy and blood results) is used, and if the variant frequency is higher than the cut-off value it is considered for further analysis. The cut-off depends on tissue quality, extraction method, biocomputing software and other parameters. Potentially causative variants need to be verified by further means (e.g. cloning + Sanger sequencing, Sanger sequencing, minisequencing).

Potentially causative variants and regions with low coverage are Sanger-sequenced. Sanger sequencing is also used for family segregation studies.

Multiplex Ligation Probe Amplification (MLPA) is used to detect duplications and deletions in ABCC6, ACVRL1, APP, CCM2, COL3A1, ENG, FBN1, GLA, KRIT1, MTHFR, NF1, NOTCH3, SAMHD1, SMAD4 and TREX1

To perform molecular diagnosis, a single sample of biological material is normally sufficient. This may be $1 \mathrm{ml}$ peripheral blood in a sterile tube with $0.5 \mathrm{ml} \mathrm{K}$ EDTA or $1 \mathrm{ml}$ saliva in a sterile tube with $0.5 \mathrm{ml}$ ethanol $95 \%$. Sampling rarely has to be repeated.

A frozen intra-lesional biopsy specimen, in addition to blood or saliva, is necessary to test for somatic variations.

Gene-disease associations and the interpretation of genetic variants are rapidly developing fields. It is therefore possible that the genes mentioned in this note may change as new scientific data is acquired. It is also possible that genetic variants today defined as of "unknown or uncertain significance" may acquire clinical importance.

\section{Genetic test results \\ Positive}

Identification of pathogenic variants in the above genes confirms the clinical diagnosis and is an indication for family studies.

A pathogenic variant is known to be causative for a given genetic disorder based on previous reports, or predicted to be causative based on loss of protein function or expected significant damage to proteins or protein/protein interactions. In this way it is possible to obtain a molecular diagnosis in new/other subjects, establish the risk of recurrence in family members and plan preventive and/or therapeutic measures.

\section{Inconclusive}

Detection of a variant of unknown or uncertain significance (VUS): a new variation without any evident pathogenic significance or a known variation with insufficient evidence (or with conflicting evidence) to indicate it is likely benign or likely pathogenic for a given genetic disorder. In these cases, it is advisa- ble to extend testing to the patient's relatives to assess variant segregation and clarify its contribution. In some cases, it could be necessary to perform further examinations/tests or to do a clinical reassessment of pathological signs.

\section{Negative}

The absence of variations in the genomic regions investigated does not exclude a clinical diagnosis but suggests the possibility of:

- alterations that cannot be identified by sequencing, such as large rearrangements that cause loss (deletion) or gain (duplication) of extended gene fragments;

- sequence variations in gene regions not investigated by this test, such as regulatory regions (5' and 3' UTR) and deep intronic regions;

- variations in other genes not investigated by the present test.

\section{Unexpected}

Unexpected results may emerge from the test, for example information regarding consanguinity, absence of family correlation or other genetically-based diseases.

\section{Risk for progeny}

If the identified pathogenic variant has autosomal dominant transmission, the probability that an affected carrier transmit the disease variant to his/her children is $50 \%$ in any pregnancy, irrespective of the sex of the child conceived.

In autosomal recessive mutations, both parents are usually healthy carriers. In this case, the probability of transmitting the disorder to the offspring is $25 \%$ in any pregnancy of the couple, irrespective of the sex of the child. An affected individual generates healthy carrier sons and daughters in all cases, except in pregnancies with a healthy carrier partner. In these cases, the risk of an affected son or daughter is $50 \%$.

In $\mathrm{X}$-linked recessive inheritance, affected males transmit the pathogenic variant to their daughters and the probability that a female carrier transmit the pathogenic variant to her offspring is $50 \%$ in any pregnancy irrespective of the sex of the child conceived. Females who inherit the pathogenic variant are carriers and usually unaffected. Males who inherit the pathogenic variant are affected.

De novo somatic variations cannot be inherited or transmitted.

In mitochondrial inheritance, variations can only be transmitted by the mother.

In paradominant inheritance, only the germline genetic variant is transmitted in an autosomal dominant fashion and the probability that carriers transmit the germline pathogenic variant to their children is $50 \%$ in any pregnancy, irrespective of the sex of the child conceived.

\section{Limits of the test}

The test is limited by current scientific knowledge regarding the gene and disease. 
Analytical sensitivity (proportion of positive tests when the genotype is truly present) and specificity (proportion of negative tests when the genotype is not present)

NGS Analytical sensitivity $>99.99 \%$, with a minimum coverage of 10X; Analytical specificity $99.99 \%$.

SANGER Analytical sensitivity $>99.99 \%$; Analytical specificity $99.99 \%$.

MLPA Analytical sensitivity $>99.99 \%$; Analytical specificity $99.99 \%$.

Clinical sensitivity (proportion of positive tests if the disease is present) and clinical specificity (proportion of negative tests if the disease is not present)

Clinical sensitivity: variations in the aforementioned genes are linked to MS, but may be individual variations (identified in one or a few families) and total epidemiological data is therefore not available. Clinical sensitivity will be estimated based on internal cases.

Clinical specificity: is estimated at approximately $99 \%$ (23).

\section{Prescription appropriateness}

The genetic test is appropriate when:

a) the patient meets the diagnostic criteria for MS;

b) the sensitivity of the test is greater than or equal to that of tests described in the literature.

\section{Clinical utility}

\begin{tabular}{|l|c|}
\hline Clinical management & Utility \\
\hline Confirmation of clinical diagnosis & Yes \\
\hline Differential diagnosis & Yes \\
\hline Couple risk assessment & Yes \\
\hline $\begin{array}{l}\text { Availability of clinical trials can be checked on-line at } \\
\text { https://clinicaltrials.gov/ }\end{array}$
\end{tabular}

\section{References}

1. WHO MONICA Project Investigators The World Health Organization MONICA Project (Monitoring trends and determinants in cardiovascular disease). J Clin Epidemiol. 1988; 41(2): 105-14.

2. Adams HP Jr, Bendixen BH, Kappelle LJ, Biller J, Love BB, Gordon $\mathrm{DL}$, Marsh EE $3^{\text {rd }}$. Classification of subtype of acute ischemic stroke. Definitions for use in a multicenter clinical trial. TOAST. Trial of Org 10172 in Acute Stroke Treatment. Stroke. 1993; 24(1): 35-41.

3. Philip M, Bath W, Lees KR. ABC of arterial and venous disease. BMJ. 2000; 320: 920-23.

4. Brass LM, Isaacsohn JL, Merikangas KR, Robinette CD. A study of twins and stroke. Stroke. 1992; 23(2): 221-23.

5. Markus HS. Unravelling the Genetics of Ischaemic Stroke. PLoS Medicine. 2010; 7(3): e1000225.

6. Tonk M, Haan J. A review of genetic causes of ischemic and hemorrhagic stroke. J Neurol Sci. 2007; 257(1-2): 273-79.

7. Kittner SJ, McCarter RJ, Sherwin RW, Sloan MA, Stern BJ, Johnson CJ, Buchholz D, Seipp MJ, Price TR. Black-white differences in stroke risk among young adults. Stroke. 1993; 24(12 Supp1): I13-I15.

8. Harmsen $P$, Wilhelmsen $L$, Jacobsson A. Stroke incidence and mortality rates 1987 to 2006 related to secular trends of cardiovascular risk factors in Gothenburg, Sweden. Stroke. 2009; 40(8): 2691-2697.

9. Rannikmäe K, Sivakumaran V, Millar H, Malik R, Anderson CD, Chong $M$, Dave T, Falcone GJ, Fernandez-Cadenas I, JimenezConde J, Lindgren A, Montaner J, O’Donnell M, Paré G, Radmanesh F, Rost NS, Slowik A, Söderholm M, Traylor M, Pulit SL, Seshadri S, Worrall BB, Woo D, Markus HS, Mitchell BD, Dichgans M, Rosand J, Sudlow CLM. Stroke Genetics Network (SiGN), METASTROKE Collaboration, and International Stroke Genetics Consortium (ISGC). COL4A2 is associated with lacunar ischemic stroke and deep ICH: Meta-analyses among 21,500 cases and 40,600 controls. Neurology. 2017; 89(17): 1829-39.

10. Thiele H, du Moulin M, Barczyk K, George C, Schwindt W, Nürnberg G, Frosch M, Kurlemann G, Roth J, Nürnberg P, Rutsch F. Cerebral arterial stenoses and stroke: novel features of Aicardi-Goutières syndrome caused by the Arg164X mutation in SAMHD1 are associated with altered cytokine expression. Hum Mutat. 2010; 31(11): E1836-50.

11. Rost NS, Greenberg SM, Rosand J. The genetic architecture of intracerebral hemorrhage. Stroke. 2008; 39(7): 2166-73.

12. Tonk M, Haan .J A review of genetic causes of ischemic and hemorrhagic stroke. J Neurol Sci. 2007; 257(1-2): 273-79.

13. Liebeskind D. Hemorrhagic Stroke. Updated: Jan 23, 2017 by WebMD LLC. available from: https://emedicine.medscape.com/ article/1916662-overview\#a5.

14. Global Burden of Stroke. The atlas of Heart Disease and Stroke. MacKay J, Mensah GA. World Health Organization. Available from: http://www.who.int/cardiovascular_diseases/en/cvd_atlas_15_ burden_stroke.pdf

15. van Asch CJ, Luitse MJ, Rinkel GJ, van der Tweel I, Algra A, Klijn CJ. Incidence, case fatality, and functional outcome of intracerebral haemorrhage over time, according to age, sex, and ethnic origin: a systematic review and meta-analysis. Lancet Neurol 2010; 9(2): 167-76.

16. Aguilar MI, Freeman WD. Spontaneous intracerebral hemorrhage. Semin Neurol 2010; 30(5): 555- 64.

17. Broderick J, Connolly S, Feldmann E, Hanley D, Kase C, Krieger D, Mayberg M, Morgenstern L, Ogilvy CS, Vespa P, Zuccarello M. American Heart Association; American Stroke Association Stroke Council; High Blood Pressure Research Council; Quality of Care and Outcomes in Research Interdisciplinary Working Group. Guidelines for the management of spontaneous intracerebral hemorrhage in adults: 2007 update: a guideline from the American Heart Association/American Stroke Association Stroke Council, High Blood Pressure Research Council, and the Quality of Care and Outcomes in Research Interdisciplinary Working Group. Stroke. 2007; 116(6): e391-13.

18. Elliott J, Smith M. The Acute Management of Intracerebral Hemorrhage: A Clinical Review. Anesthesia \& Analgesia. 2010; 110(5): 1419-27.

19. Smith SD, Eskey CJ. Hemorrhagic stroke. Radiol Clin North Am. 2011; 49(1): 27-45.

20. Anderson CS, Huang $Y$, Wang JG, Arima H, Neal B, Peng B, Heeley E, Skulina C, Parsons MW, Kim JS, Tao QL, Li YC, Jiang JD, Tai LW, Zhang JL, Xu E,Cheng Y, Heritier S, Morgenstern LB, Chalmers J; INTERACT Investigators. INTERACT Investigators. Intensive blood pressure reduction in acute cerebral haemorrhage trial (INTERACT): a randomised pilot trial. Lancet Neurol. 2008; 7(5): 391-99

21. Falcone GJ, Woo D. Genetics of Spontaneous Intracerebral Hemorrhage. Stroke. 2017; 48(12): 3420-24.

22. French $C R$, Seshadri $S$, Destefano AL, Fornage $M$, Arnold CR, Gage PJ, Skarie JM, Dobyns WB, Millen KJ, Liu T, Dietz W, Kume T, Hofker M, Emery DJ,Childs SJ, Waskiewicz AJ, Lehmann OJ. Mutation of FOXC1 and PITX2 induces cerebra small-vessel disease. J Clin Invest. 2014; 124(11): 4877-81.

23. Chen B, Gagnon M, Shahangian S, Anderson NL, Howerton DA, Boone JD; Centers for Disease Control and Prevention (CDC). Good Laboratory Practices for Molecular Genetic Testing for Heritable Diseases and Conditions. MMWR Recomm Rep 2009; 58(RR6): 1-37. 